\title{
Die Rolle der Spongiosaplastik und anderer augmentierender Verfahren
}

\author{
Jörg Schmidt, Wolfgang Schnorr
}

\section{Zusammenfassung}

Die Spongiosaplastik ist heute noch immer der Goldstandard in der Augmentation von Knochendefekten am distalen Radius. Sie wird alleinstehend oder in Kombination mit anderen, augmentierenden Osteosyntheseverfahren angewendet. Anders als in der Kieferchirurgie, wo die Anwendung von Knochenersatzmaterialien breiten Einzug gehalten hat, liegen bei der distalen Radiusfraktur nur beschränkte Erfahrungen vor. Hier ist allerdings ein breites Feld für weitere, sicher erfolgreiche Forschung eröffnet.

\section{Einleitung}

Durch die zunehmende Versorgung der distalen Radiusfraktur mit winkelstabilen Implantaten hat die Notwendigkeit, Spongiosaplastiken oder andere augmentierende Verfahren am distalen Radius zu verwenden, abgenommen.

Bei den 23 A3-Frakturen, den dorsal eingestauchten distalen Radiusfrakturen mit dorsaler Trümmerzone, kommt es durch die Aufrichtung des Bruches und die winkelstabile Versorgung zwar zu einem streckseitig knöchernen Defekt, durch die Winkelstabilität ist jedoch trotzdem eine für die funktionelle Nachbehandlung notwendige Stabilität der Versorgung gegeben. Trotzdem gibt es noch immer Frakturen, z.B. bei den gelenkbildenden Frakturen der Klassifikation 23 C 2 und C 3, die durch winkelstabile Implantate

OP-JOURNAL 2003; 19: 52-55

(c) Georg Thieme Verlag Stuttgart · New York eben zu versorgen sind. Hier besteht noch immer die Notwendigkeit, die entspr. Hohlräume auszufüllen.

\section{Indikation des Knochenersatzes}

Gelingt eine winkelstabile Versorgung einer distalen Radiusfraktur nicht und muss diese mit einem Fixateur externe behandelt werden, so ist bekannt, dass diese Frakturen trotz der Ausnutzung der Ligamentotaxis in der Regel dorsalaxial einsintern. Dies führt wieder zu einem relativen Ulnavorschub und damit zu der bekannten Problematik des ulnaren Handgelenkkompartimentes.

Um dieses dorso-axiale Zusammensintern zu verhindern, müssen diese durch Spongiosakompression und Reposition entstandenen Defekte der Radiusepiphyse aufgefüllt werden $[1,7,9,10,17]$.

Schwierig zu behandeln wird eine Fraktur dann, wenn das sogen. ulnare Schlüsselfragment axial eingebrochen ist. Diese ulnare Kante des distalen Radius trägt das Lunatum und ist damit in der Hauptbelastungsachse des Handgelenkes gelegen (Abb.1). Sie hat keine Bandverbindung und ist damit durch die Ligamentotaxis weder reponierbar noch retinierbar.

Bei fehlender Ligamentotaxis und Defekt kommt es immer zu einer Redislokation !

Dieses Fragment muss aktiv aufgerichtet und abgestützt werden. Dies gelingt am besten durch Auffüllen des darunter entstandenen Hohlraumes und Defektes mit einem Knochenersatz (Abb. 2).

Eine weitere Indikation zum Knochenersatz bei der distalen Radiusfraktur ist die sehr selten auftretende, dann aber schwierig zu behandelnde Pseudarthrose des distalen Radius. Hier muss nach stabiler Osteosynthese „Biologie“, also wachstumsaktives Material, in die Pseudarthrose eingebracht werden.

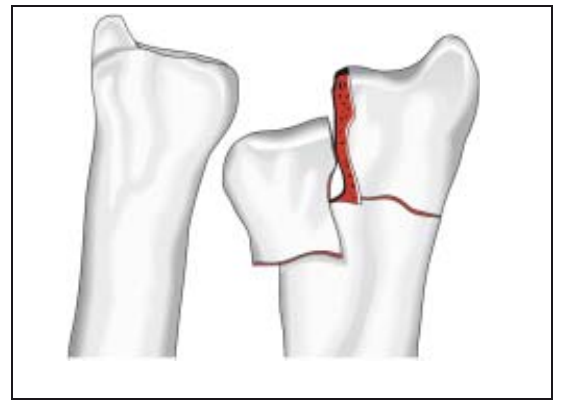

Abb.1 Eingebrochenes ulnares Schlüsselfragment.

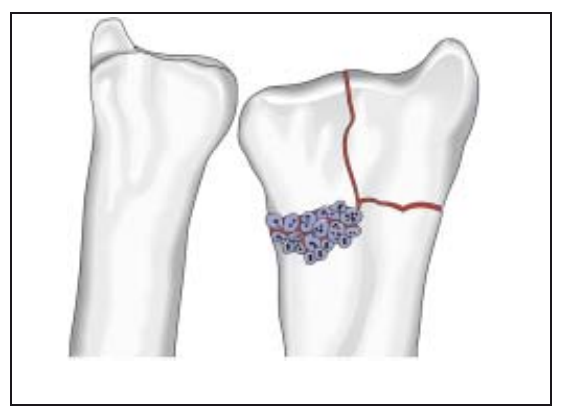

Abb. 2 Prinzip der Operation - Ausfüllen entstandener Hohlräume.

Eine dritte Indikation ist die Umstellungsosteotomie, welche jedoch an anderer Stelle in diesem Heft beschrieben wird.

\section{Knochenersatzmaterialien}

Eine ganze Ansammlung von verschiedenen Knochenersatzmaterialien wird mittlerweile auf dem Markt angeboten.

Die Knochenersatzmaterialien werden ganz grob unterteilt in:

a) osteokonduktive Materialien: dienen als biologische Leitschiene für Knochenwachstum

b) osteoinduktive Materialien: werden in den neugebildeten Knochen ein- und später abgebaut

c) osteointegrative Materialien, die durch Besetzung mit Wachstumsfaktoren in 
den neugebildeten Knochen integral eingebaut werden.

Rüger [12] hat 1998 versucht, mit einer Klassifikation eine Systematik in die Knochenersatzmaterialien zu bringen.

Beschrieben wurden 4 Klassen:

\section{Klasse 1 - biologisch-organische} Substanzen,

a) Knochenmatrices

b) Knochenmatrixextrakte

c) extrahierte Knochenwachstumsfaktoren

d) nicht knochenspezifische Wachstumsfaktoren

Klasse 2 - synthetische, anorganische Materialien

a) monophasisch-synthetische Verbindungen

b) Hydrothermal aus Kollagen bzw. Algen gewonnene Verbindungen

c) aus Rinderknochen gewonnene Verbindungen

d) mehrphasische Kaliziumphosphatkeramiken, Biogläser oder mehrphasige Glaskeramiken

e) kalziumphosphathaltige Knochenzemente

Klasse 3 - synthetische, organische Verbindungen

a) Polyester

b) Polyaminosäuren

c) Polyanhydride

d) Polyorthoesther

e) Polyphosphazene

\section{Klasse 4 - Komposite}

z.B. Metallspongiosa mit autogenem Knochen oder

synthetisches Hydroxylapatit in Verbindung mit Rinderkollagen

Allein aufgrund dieser Aufzählung ist zu sehen, dass auf vielen Gebieten der Knochenersatzmaterialien geforscht wird.

4 Klassen der Knochenersatzmaterialien: 1. biologisch organische Substanzen

2. synthetisch anorganische Substanzen

3. synthetisch organische Verbindungen

4. Komposite

Käuflich zu erwerben sind zum einen kalziumphosphathaltige Knochenzemente (z.B. Norian SRS ${ }^{\circledR}$ ) oder durch thermische
Sinterungsprozesse gewonnene Materialien aus Rinderknochen, (z.B. Bio-Oss ${ }^{\circledR}$ ), synthetische Hydroxyepatitkeramiken (z.B.Syntharcer ${ }^{\circledR}$ ) oder reine Betatrikalziumphosphate (z.B. chronOS $^{\circledR}$ ).

Das Einbringen von Knochen- oder Gewebewachstumsfaktoren (BMP, FGF usw.) hat in experimentellen Studien bisher kein besseres Einwachsen von Knochenersatzmaterialien in der Spenderregion zeigen können [13].

Verschiedene experimentelle Studien haben zwar gezeigt, dass das Kalluswachstum in der Umgebungsreaktion bei der Anwesenheit von BMPs deutlich erhöht wird, dass jedoch ein Einwachsen der Knochenersatzmaterialien in der Spenderregion dadurch nicht verbessert wird [13]. Auch hier besteht noch Handlungsbedarf.

Experimentiert wird vor allem mit biologischen Knochenersatzmaterialien, bei denen auf z.B. kollagenen Fasern körpereigene Osteoblasten angesiedelt werden in der Hoffnung, hier eine höhere Dichte an körpereigenen Osteoblasten im Defekt zu erreichen.

Die Vorteile der Knochenersatzmaterialien liegen in ihrer unbegrenzten Verfügbarkeit und insbesondere in dem fehlenden Hebedefekt beim Patienten $[15,16]$.

Allgemein ist bekannt, dass der Hebedefekt am Beckenkamm bei Patienten mit distaler Radiusfraktur oftmals länger und mehr schmerzt als die Empfängerregion am distalen Handgelenk und der Pat. schon eine passable Beweglichkeit des Handgelenkes aufweist, aber noch ein hinkendes Gangbild zeigt.

Diesem Vorteil stehen natürlich gewisse Nachteile entgegen.

Alle biologischen Ersatzmaterialien sind zum jetzigen Zeitpunkt noch teuer und belasten dabei erheblich das Budget der unfallchirurgischen Kliniken.

In Zeiten der BSE-Krise sah man, dass auch aufbereitete Rindermaterialien durchaus für den Menschen Gefährdungen darstellen können.

In letzter Zeit haben Studien gezeigt, dass wie bei allen Fremdmaterialien, die implantiert werden, auch Knochenersatzmaterialien eine pathologische Bakterienanhaftung aufweisen und somit mutmaßlich, wie alle anderen Implantate auch, Ausgangspunkt eines chronischen Infektes darstellen können [6].

Knochenersatzmaterialien

Vorteile:

- Verfügbarkeit

kein Hebedefekt

Nachteile:

a teuer

Keimbesiedelung (?)

- Einwachsverhalten

\section{Autologe Spongiosaplastik}

Die autologe Spongiosaplastik ist bei Knochendefekten zum jetzigen Zeitpunkt noch immer der „Goldstandard“. Als Knochenspender dient in der Regel der vordere oder hintere Beckenkamm. Diese Beckenkammspongiosa hat die höchste osteogene Potenz aller Spenderregionen des menschlichen Körpers. Weiterhin können verwendet werden: der Tibiakopf, die Trochanterregion des Femurs, der distale Radius bei kleinen Mengen.

Der Vorteil der autologen Spongiosaplastik liegt vor allem darin, dass es sich um ein körpereigenes Material handelt. Die immunologische und osteogene Potenz ist trotz aller Versuche mit Wachstumsfaktoren noch immer ungleich höher als bei Ersatzmaterialien. Spongiosa kostet nichts außer einem etwas erweiterten operativen Aufwand. Der Hauptvorteil liegt in der schnellen Integration des $\mathrm{Er}$ satzmaterials in dem knöchernen Defekt.

Nachteile der Spongiosaplastik liegen in dem zweiten chirurgischen Eingriff mit allen Komplikationsmöglichkeiten und in der doch begrenzten Verfügbarkeit vor allem bei Zweiteingriffen.

Zum jetzigen Zeitpunkt ist nach Abwägung aller Vor- und Nachteile die autologe Spongiosaplastik bei der distalen Radiusfraktur noch immer der Goldstandard und bringt die schnellste Einheilung des knöchernen Aufbaus [1,2,7,9,10,17].

Autologe Spongiosa

Vorteile:

cörpereigen

schnelle Integration

- eigene „Biologie“

Nachteile:

2. Eingriff

begrenzt verfügbar 


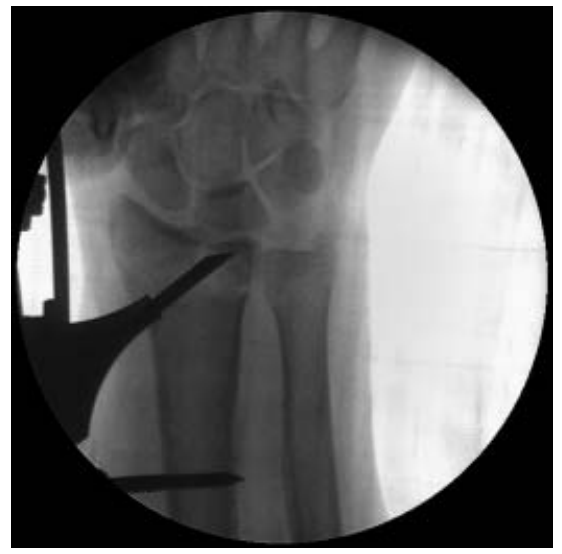

Abb. 3 1. OP-Schritt - Reposition.

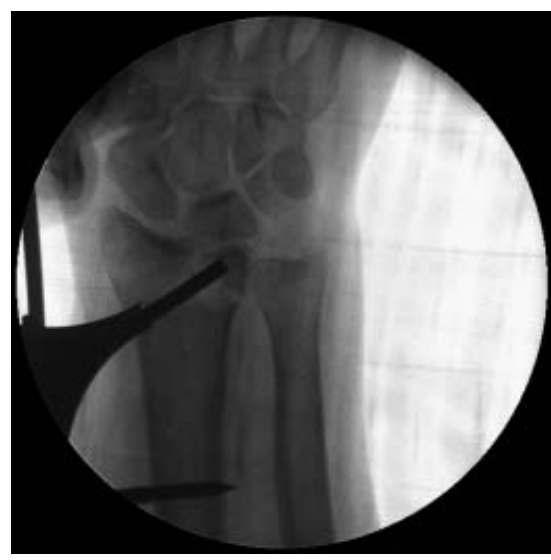

Abb. 4 2. OP-Schritt - Einbringen und Verdichten des Knochenersatzes.

\section{Technik der minimalinvasiven Spongiosaplastik}

Über einen maximal $1 \mathrm{~cm}$ langen Zugang streng radial kann die Frakturzone eröffnet und ein Spongiosatrichter eingebracht werden. Verschiedene Stößel ermöglichen die Reposition gegebenenfalls dislozierter oder eingestauchter Fragmente (Abb.3). Die ebenfalls minimalinvasiv aus dem Beckenkamm oder Tibiakopf gewonnene Spongiosa kann mit den Instrumenten gezielt unter die dislokationsgefährdeten Areale gebracht werden (Abb.4).

Zur Durchführung der minimalinvasiven Spongiosaplastik wurde ein Spezialinstrumentarium entwickelt (MINISPONG).

Dieses setzt sich zusammen aus einem Spongiosatrichter mit $7 \mathrm{~mm}$ Außendurchmesser und $5 \mathrm{~mm}$ Innendurchmesser. Zum Einbringen des Trichters und Eröffnen der Frakturzone benutzt man

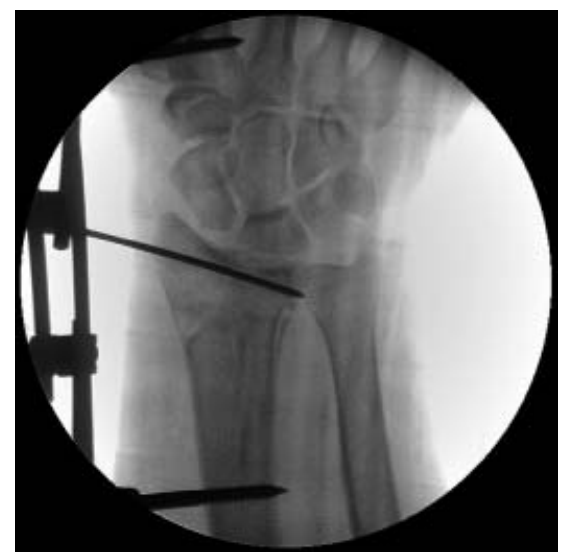

Abb. 5 3. OP-Schritt - Zusätzliche Implantataugmentation.

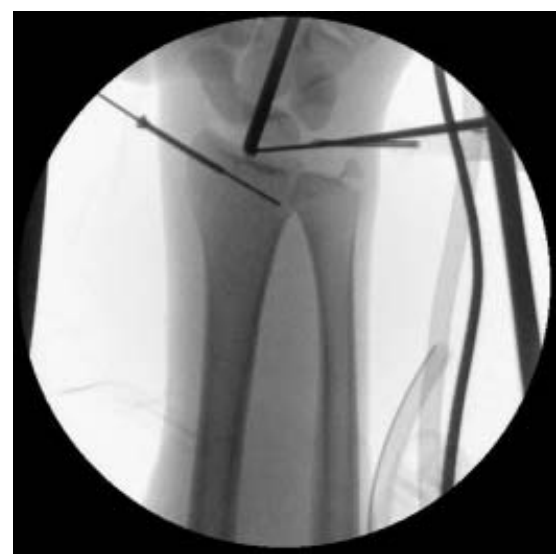

Abb. 6 Arthroskopische Kontrolle des Operationserfolges.

einen $5 \mathrm{~mm}$ Eröffnungspfriem mit scharf geschliffener Spitze.

Der Zugang ist streng radial lateral, dorsal des Verlaufes des n. radialis superficialis. Eine $1 \mathrm{~cm}$ lange, in Längsachse des Unterarmes verlaufende Inzision reicht aus. Diese wird stumpf bis auf den Knochen gespreizt. Unter dem Schutz der geöffneten Klemme perforiert der Eröffnungspfriem die laterale Kortikalis, über den Pfriem wird der Trichter eingeführt.

Zur Reposition einzelner Fragmente oder der Auffüllung von Hohlräumen, die Fraktur ist mit dem Fixateur externe bereits reponiert, stehen verschiedene Stößel zur Verfügung. Ein gebogener und ein abgeschrägter Stößel mit 3 mm Durchmesser dienen zur Reposition und zum gezielten Aufstößeln imprimierter Fragmente. Ein Verdichterstößel mit $5 \mathrm{~mm}$ Außendurchmesser dient zum Einbringen und Verdichten der Spongiosa.

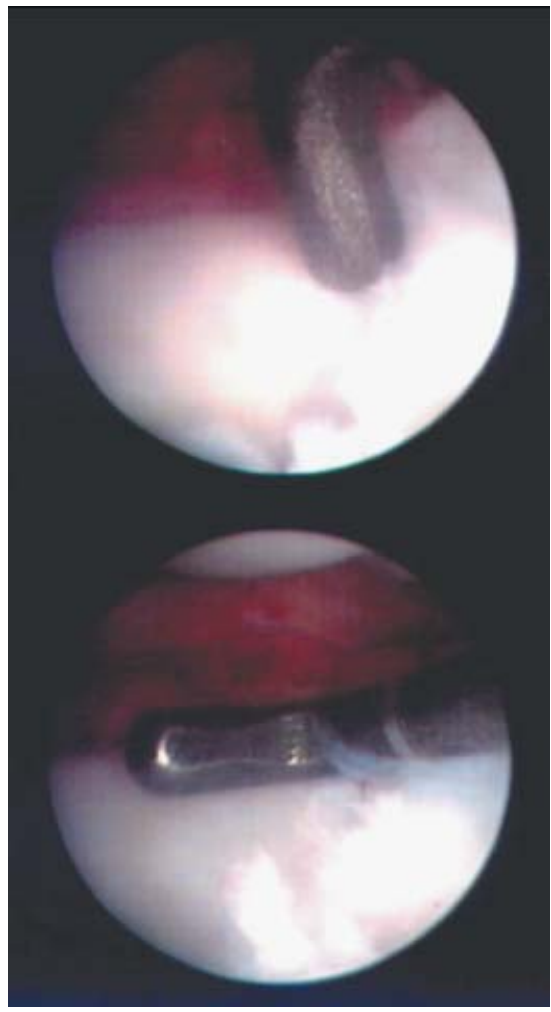

Abb. 7 Arthroskopisches Bild.

Zur Kontrolle der Fragmentstellung und der Lage der Spongiosaplastik wird der Bildwandler oder das Arthroskop genutzt (Abb.6 u. 7).

\section{Weitere augmentierende Verfahren}

Nicht nur Knochen oder Knochenersatz werden zum Redislokationsschutz von distalen Radiusfrakturen benutzt. Auch zusätzlich zum eigentlichen Osteosyntheseverfahren eingebrachte KirschnerDrähte oder Schrauben sind als augmentierende Verfahren zu werten. Insbesondere bei C3-Frakturen ist der Fixateur externe oftmals als alleiniges Verfahren überfordert und muss unterstützt werden (Abb. 5 u. 6).

Auch zusätzlich eingebrachte Implantate sind augmentierende Maßnahmen!

\section{Literatur}

${ }^{1}$ Barbieri CH, Mazzer N, Aranda CA, Pinto MM. Use of a bone block graft from the iliac crest with rigid fixation to correct diaphyseal defects of the radius and ulna. J Hand Surg 1997; 22(3): 395-401

${ }^{2}$ Cannegieter DM, Juttmann JW. Cancellous grafting and external fixation for unstable Colles' fractures. J Bone Joint Surg Br 1997; 79(3): 428-432 
${ }^{3}$ Chen AC, Chan YS, Yuan LJ, Ye WL, Lee MS, Chao EK. Arthroscopically assisted osteosynthesis of complex intra-articular fractures of the distal radius. J Trauma 2002; 53(2): 354-359

${ }^{4}$ Fernandez DL, Mader K. The treatment of complex carpal dislocations by external fixation. Injury 2000; 31: $92-101$

${ }^{5}$ Gausepohl T, Pennig D, Mader K. Principles of external fixation and supplementary techniques in distal radius fractures. Injury 2000; 31: 56-70

${ }^{6}$ Geyer G, Schott C, Schwarzkopf A. Effects of alloplastic bone substitutes on bacterial growth HNO 1999; 47: 25-32

7 Herrera M, Chapman CB, Roh M, Strauch RJ, Rosenwasser MP. Treatment of unstable distal radius fractures with cancellous allograft and external fixation. J Hand Surg [Am] 1999; 24(6): 1269-1278

${ }^{8}$ Klein W, Dee W, Rieger H, Neumann H, Joosten U. Results of transarticular fixator application in distal radius fractures. Injury 2000; 31: $71-77$

${ }^{9}$ Ladd AL, Pliam NB. The role of bone graft and alternatives in unstable distal radius fracture treatment. Orthop Clin North Am 2001; 32(2): $337-351$

${ }^{10}$ Ladd AL, Pliam NB. Use of bone-graft substitutes in distal radius fractures. J Am Acad Orthop Surg 1999; 7(5): 279-290
${ }^{11}$ Ring D, Roberge C, Morgan T, Jupiter JB. Osteotomy for malunited fractures of the distal radius: a comparison of structural and nonstructural autogenous bone grafts. J Hand Surg [Am] 2002; 27(2): 216-222

${ }^{12}$ Rueger JM. Bone replacement materials state of the art and the way ahead. Orthopäde 1998; 27: $72-79$

${ }^{13}$ Schnettler R, Markgraf E. (Hrsg.) Knochenersatzmaterialien und Wachstumsfaktoren. Georg Thieme Verlag Stuttgart - New York 1997

${ }^{14}$ Wahlenkamp GHIM. Biomaterials in Surgery. Georg Thieme Verlag Stuttgart - New York 1998

${ }^{15}$ Werber KD, Brauer RB, Weiss W, Becker K. Osseous integration of bovine hydroxyapatite ceramic in metaphyseal bone defects of the distal radius. J Hand Surg [Am] 2000; 25(5): 833-841

${ }^{16}$ Wolfe SW, Pike L, Slade JF 3rd, Katz LD. Augmentation of distal radius fracture fixation with coralline hydroxyapatite bone graft substitute. J Hand Surg [Am] 1999; 24(4): $816-827$

${ }^{17}$ Wright RR, Schmeling GJ, Schwab JP. The necessity of acute bone grafting in diaphyseal forearm fractures: a retrospective review. J Orthop Trauma 1997; 11(4): $288-294$

\section{Dr. med. Jörg Schmidt}

Chefarzt

Dr. med. Wolfgang Schnorr

Ltd. Oberarzt

Unfallchirurgische Klinik am HELIOS Klinikum Berlin Hobrechtsfelder Chaussee 100 D-13125 Berlin 